\title{
Development of a nano-modified glassy carbon electrode for the determination of 2,6-diaminotoluene (TDA)
}

\author{
Duygu Büyüktaş $^{\mathrm{a}}$, Masoud Ghaani ${ }^{\mathrm{b}}$, Cesare Rovera ${ }^{\mathrm{b}}$, Richard T. Olsson ${ }^{\mathrm{c}}$, Figen Korel ${ }^{\mathrm{a}}$, \\ Stefano Farris ${ }^{\mathrm{b}, \mathrm{d}, *}$ \\ ${ }^{a}$ Department of Food Engineering, Faculty of Engineering, Izmir Institute of Technology, 35430, Gülbahçe Köyü, Urla, Izmir, Turkey \\ ${ }^{\mathrm{b}}$ DeFENS, Department of Food, Environmental and Nutritional Sciences, Food Packaging Lab., University of Milan, via Celoria 2 - I, 20133, Milan, Italy \\ ${ }^{\mathrm{c}}$ Department of Fibre and Polymer Technology, School of Chemical Science and Engineering, KTH Royal Institute of Technology, Teknikringen 56, SE-100 44, Stockholm, \\ Sweden \\ d INSTM, National Consortium of Materials Science and Technology, Local Unit University of Milan, via Celoria 2 - I, 20133, Milan, Italy
}

\section{A R T I C L E I N F O}

\section{Keywords:}

Electrochemical sensor

Food packaging

Primary aromatic amines

Multi-walled carbon nanotube

Mesoporous carbon nanoparticles

Migration

\begin{abstract}
A B S T R A C T
The objective of this study was to improve the overall performance of a glassy carbon electrode (GCE) for the detection of 2,6-diaminotoluene (TDA), a possibly carcinogenic primary aromatic amines (PAAs) that poses a serious risk for the consumer' health because they can transfer from multilayer food packages including adhesives based on aromatic polyurethane (PU) systems, to the food. The modification of the electrode surface was made by means of multi-walled carbon nanotubes (MWCNTs) and mesoporous carbon nanoparticles (MCNs). The MWCNTs-MCNs/GCE allowed achieving the best performance in terms of sensitivity, as revealed by cyclic voltammetry $-\mathrm{CV}$, with an oxidation peak of $20.95 \mu \mathrm{A}$ over $0.079 \mu \mathrm{A}$ of the bare GCE. The pH of the medium influenced the oxidation of 2,6-TDA, with highest sensitivity at $\mathrm{pH} \sim 7$. Amperometry experiments led to an estimated detection limit of $0.129 \mu \mathrm{M}$, and three linear ranges were obtained for 2,6-TDA: 0.53-11.37 $\mu \mathrm{M}$, 11.37-229.36 $\mu \mathrm{M}$, and 229.36-2326.60 $\mu \mathrm{M}$. Chronoamperometry experiments combined with Cottrell's theory allowed estimating a diffusion coefficient of 2,6-TDA of $1.34 \times 10^{-4} \mathrm{~cm}^{2} \mathrm{~s}^{-1}$. The number of electrons $(n \sim 1)$ involved in the catalytic oxidation of 2,6-TDA was determined according to the Laviron's theory. Real sample tests demonstrated that the modification of the sensor using nanoparticls allowed to obtain a highly sensitive and selective sensor, which can possibly used as an alternative analytical device for the rapid, easy, and reliable determination of 2,6-TDA.
\end{abstract}

\section{Introduction}

Multilayer materials represent the first-choice option for many different food applications because they allow to achieve an outstanding overall performance by combining the properties of each individual, such as printability, barrier properties against gases and vapors, mechanical performance, and thermal properties (e.g., sealability) (Goulas, Riganakos, \& Kontominas, 2003). Multilayers materials are manufactured by the converting industry, which deals with processing operations (such as printing, coating, and laminating) to convert the pristine flat web into an intermediate form or the final packaging material. Among the wide variety of adhesives, polyurethane (PU) adhesives are the most extensively used by the converting industry owing to their unrivaled performance, as demonstrated by their largest market share in food-contact adhesives (Yan, Hu, Tong, Lei, \& Lin, 2020). However, a risk associated with PU adhesives is the possible formation of undesired by-products, also defined as non-intentionally added substances (NIAS), such as cyclic polyesters oligomers (Ubeda, Aznar, Rosenmai, Vinggaard, \& Nerín, 2020) primary aromatic amines (PAAs). The origin of PAAs, while primarily linked to residual (unreacted) isocyanic monomers, has more recently been ascribed to the cleavage of secondary bonds on the main PU backbone (e.g., biuret and especially allophanate bonds) due to thermal stresses, such as those represented by conventional thermal operations (e.g., pasteurization and sterilization) and cooking methods (e.g., vacuum-cooking, microwaving, etc.) (Campanella, Ghaani, Quetti, \& Farris, 2015). In spite of the large number of

\footnotetext{
* Corresponding author at: DeFENS, Department of Food, Environmental and Nutritional Sciences, Food Packaging Lab., University of Milan, via Celoria 2 - I, 20133, Milan, Italy.

E-mail address: stefano.farris@unimi.it (S. Farris).
} 
PAAs, those of relevance for the food packaging sector are the 4 , 4'-methylene diphenyl diamine, the 2,6-diaminotoluene (2,6-TDA), and the 2,4-diaminotoluene (2,4-TDA), which arise from the methylene diphenyl diisocyanate (MDI) and toluene diisocyanate (TDI) isocyanic monomers. These monomers account for almost the totality of isocyanic monomers used for the obtainment of aromatic PU adhesive systems. TDI monomers, in particular, find predominant use in the manufacturing of solvent-based $\mathrm{OH}$-terminated PU adhesives. In this case, a mixture of the two isomers (2,6-toluene diisocyanate and 2,4-toluene diisocyanate) is most often used.

Possible contamination of food by PAAs poses a serious threat to the consumers' health because PAAs have been defined as "possibly carcinogenic to humans" (Vineis \& Pirastu, 1997). In contrast to the U.S., where the FDA has forbidden the use of aromatic-based PU adhesives, European Union (EU) legislation allows their use while setting a specific migration limit of PAAs to food as $10 \mathrm{ng}$ for total PAAs to protect consumers' health (European Commission, 2011; Mortensen, Trier, Foverskov, \& Petersen, 2005). From a practical point of view, the detection of PAAs occurs mainly using the spectrophotometric method developed by the German Federal Institute for Health Protection of Consumers and Veterinary Medicine (BgVV) (Brauer \& Funke, 1991). According to this method, quantification of PAAs is expressed as equivalent to aniline. The main drawback associated with this method is its non-selectivity, which may lead to overestimate the ultimate quantification of PAAs in food simulants (Aznar, Canellas, \& Nerín, 2009; Brede \& Skjevrak, 2004). For achieving a selective detection of PAAs, other analytical techniques have been proposed as alternatives to the spectrophotometric method. Among others, chromatography (Cai, Ge, Ouyang, Hu, \& Li, 2020), mass spectrometry (Sanchis, Coscollà, \& Yusà, 2019), capillary electrophoresis (Mattarozzi, Lambertini, Suman, \& Careri, 2013), and electrochemical methods (Chen et al., 2015) proved to be effective for the detection of trace amounts of PAAs transferred from the packaging material. However, also the above methods have drawbacks. For example, they are complex and time consuming especially as far as the sample preparation is concerned. In addition, the high cost of the necessary equipment, together with the necessity of specialized operators, makes these methods unaffordable by most players of the converting industry (Ghaani, Pucillo, Olsson, Scampicchio, \& Farris, 2018; Ghaani, Rovera et al., 2018). For these reasons, new analytical tools that are selective, sensitive, easy to use, and inexpensive for the determination of PAAs may represent an important advancement in the food industry, especially for quality control purposes (Ghaani, Pucillo et al., 2018; Viswanathan \& Radecki, 2008).

In recent years, electrochemical methods have attracted much attention as reliable analytical devices because they proved to possess all the aforementioned attributes. However, electrochemical sensors do not always exhibit adequate performance especially in terms of selectivity, due to the similar chemical structures of PAAs (Chen et al., 2015). To improve the electrode's overall performance, the surface of the electrode can be modified using nanoparticles, such as carbon-based nanomaterials and metallic nanoparticles (Rassaei et al., 2011; Wang et al., 2014; Wang et al., 2015; Zhang, Xing, Pan, Wang, \& Liu, 2020). These nanosized entities help, for example, prevent fouling and increase the selectivity and electrocatalytic properties of the sensor owing to large surface area and high electrical conductivity (Hanssen, Siraj, \& Wong, 2016).

In this work, we described the development of a novel selective electrochemical sensor modified with carbon nanotubes (MWCNTs) and mesoporous carbon nanoparticles (MCNs) for the quantification of 2,6diaminotoluene (2,6 TDA), which is a typical PAAs that can transfer from PU adhesives across the packaging thickness, eventually contaminating the food surface. The modified electrochemical sensor was deeply characterized as far as its electrochemical properties were concerned. The sensor modification that exploited the synergism between MWCNTs and MCNs led to a high selective, high sensitive, and with a lower limit of detection modified MWCNT-MCN/GCE, in addition to the prevention of fouling on the electrode surface. Finally, the analytical behavior of the sensor was assessed simulating a real-case scenario using a typical packaging configuration.

\section{Materials and methods}

\subsection{Reagents and equipment}

2,6-TDA (analytical grade $98 \%$, molar mass $122.17 \mathrm{~g} \mathrm{~mol}^{-1}$ ) and MCNs (500 nm particle size) were bought from Sigma Aldrich (Milan, Italy). All the other chemicals and reagents were the same as in our previous work (Ghaani, Pucillo et al., 2018; Ghaani, Rovera et al., 2018). Britton-Robinson (B-R) universal buffer $(0.04 \mathrm{M}$ boric acid, $0.04 \mathrm{M}$ acetic acid and $0.04 \mathrm{M}$ phosphoric acid) was prepared in deionized water and was tested as the supporting electrolyte.

Electrochemical experiments were performed using a PGSTAT $302 \mathrm{~N}$ potentiostat (Metrohm, Herisau, Switzerland) equipped with a threeelectrode electrochemical cell on which was mounted a modified glassy carbon (working) electrode, a platinum (counter/auxiliary) electrode, and an $\mathrm{Ag} / \mathrm{AgCl}$ (reference) electrode saturated with $3 \mathrm{M} \mathrm{KCl}$, all immersed in a double-jacket $80 \mathrm{~mL}$ glass cell (Bio-Logic, Claix, France). The software Nova 2.0 was used throughout the electrochemical experiments. The $\mathrm{pH}$ measurements were performed with a BASIC $20+$ pH meter (Crison Instruments, S.A. Barcelona, Spain). All the experiments were carried out at $25 \pm 0.5^{\circ} \mathrm{C}$.

\subsection{Electrodes modification}

A fine dispersion of MWCNTs $(0.5 \mathrm{mg}$ ) in ethylene glycol monomethyl ether (EGMe) $(1.0 \mathrm{~g}$ ) was prepared using an ultrasonicator (mod. UP400S, Hielscher, Teltow, Germany) fitted with a H3 sonotrode with a conical geometry. The ultrasonication process was performed according to the following setup: 0.5 cycle and $50 \%$ amplitude for $10 \mathrm{~min}$. The same procedure was used to obtain a dispersion of MCNs $(0.15 \mathrm{mg})$ in EGMe $(1.0 \mathrm{~g})$. The MWCNT-EGMe solution $(600 \mu \mathrm{L})$ and the MCN-EGMe solution $(150 \mu \mathrm{L})$ were then mixed by an additional ultrasonication cycle (3 $\mathrm{min})$.

Modification of the bare GCE surface was anticipated by mechanical polishing with alumina powder. Three different modifications of the GCE surface were obtained using the MWCNTs dispersion, the MCNs dispersion, and the mixture thereof, so that the MWCNTs/GCE, the MCNs/GCE, and the MWCNT-MCN/GCE modifications were obtained. For all the three modifications, $25 \mu \mathrm{L}$ of the fine dispersion were displaced on the bare electrode surface. Drying of the coating was performed using an IR lamp (type B, $1440 \mathrm{~W}$, Helios Italquartz srl, Cambiago, Italy) placed $40 \mathrm{~cm}$ away from the GCE surface throughout $10 \mathrm{~min}$. The electrode surface was finally rinsed with double-distilled water and stored at $4{ }^{\circ} \mathrm{C}$ before usage.

The effective surface area of the MWCNT-MCN/GCE was estimated from the cyclic voltammograms of $1.0 \mathrm{mM} \mathrm{K}_{3}\left[\mathrm{Fe}(\mathrm{CN})_{6}\right]$ solution at various scan rates. For a reversible process, the Randles-Sevcik formula was used (Bard \& Faulkner, 2001; Nasirizadeh et al., 2011).

$I_{p a}=2.69 \times 10^{5} n^{3 / 2} A C_{O} D^{1 / 2} v^{1 / 2}$

where $I_{p a}$ refers to the anodic peak current, $n$ the number of electrons transferred, $A$ the surface area of the electrode, $D$ the diffusion coefficient, $C_{O}$ the concentration of $\mathrm{K}_{3}\left[\mathrm{Fe}(\mathrm{CN})_{6}\right]$, and $\nu$ is the scan rate. For 1.0 $\mathrm{mM} \mathrm{K}_{3}\left[\mathrm{Fe}(\mathrm{CN})_{6}\right]$ in the $0.1 \mathrm{M} \mathrm{KNO}_{3}$ electrolyte: $n=1$ and $D=7.6 \times$ $10^{-6} \mathrm{~cm}^{2} \mathrm{~s}^{-1}$ (Bard \& Faulkner, 2001; Nasirizadeh, Shekari, Zare, \& Makarem, 2013), then from the slope of the $I_{p a}$ versus $v^{1 / 2}$, the effective areas of MWCNT-MCN/GCE was calculated as $0.0653 \mathrm{~cm}^{2}$.

\subsection{Electrode surface morphology}

Morphological views of the electrodes were obtained using a Hitachi 
S-4800 (Schaumburg, IL) field emission scanning electron microscope (FE-SEM). To this scope, MWCNT/GCE, MCN/GCE, and MWCNT-MCN/ GCE surfaces were first sputtered with Pt/Pd (60/40) for $20 \mathrm{~s}$ at a current of $80 \mathrm{~mA}$ under an argon atmosphere. Images acquisition was carried out at an acceleration voltage of $1-5 \mathrm{kV}$ and an electrode current of $10 \mu \mathrm{A}$.

\subsection{Real sample tests}

The assessment of the modified electrode performance in a real scenario was performed according to the method reported elsewhere (Ghaani, Pucillo et al., 2018). Briefly, three-layer pouches $\left(2 \mathrm{dm}^{2}\right.$ surface area) made of polyethylene terephthalate (PET, $12 \mu \mathrm{m}$ thick), polyvinilidene chloride coating (PVDC, $6 \mu \mathrm{m}$ thick), and low-density polyethylene (LDPE, $50 \mu \mathrm{m}$ thick), including a PU adhesive, were filled with $100 \mathrm{~mL}$ of an acetic acid ( $3 \mathrm{w} / \mathrm{v} \%)$ water solution (simulant B). The choice of simulant B accounts for the worst scenario for a possible transfer of PAAs from multilayer packaging materials that include a PU adhesive system. The test was carried out according to a conventional sterilization protocol, that is, at $121{ }^{\circ} \mathrm{C}$ for 20 min using an Asal 760 autoclave (Steroglass srl, Perugia, Italy). These conditions were selected with the goal of assessing the effect of high thermal treatments (e.g., pasteurization and sterilization), which have been recently indicated as one potential causes of PAAs formation due to the cleavage of secondary bonds on the main PU backbone (e.g., biuret and especially allophanate bonds) (Campanella et al., 2015).

After completion of the sterilization, specific amounts of 2,6-TDA were added in a $20 \mathrm{~mL}$ simulant B / PBS solution (1:1) and detected by amperometry, which eventually yielded the final recovery (\%) of the analyte.

\section{Results and discussion}

\subsection{Morphological characterization of modified GCEs}

Fig. 1 displays FE-SEM micrographs of the modified surface of the electrochemical sensors. The modification with MWCNTs yielded a rough morphology (Fig. 1A) caused by individual and clustered carbon nanotubes (inset of Fig. 1A). After modification of the bare GCE with MCNs, spherical particles of $80-100 \mathrm{~nm}$ diameter were observed (Fig. 1B). When the electrode was modified with MWCNTs and MCNs, MWCNTs appeared immersed the spherical domains (Fig. 1C), with some individual nanotubes pointing out to the medium. (inset of Fig. 1C).

\subsection{Electrochemical behavior of the modified electrodes}

Cyclic voltammetry (CV) was used to investigate the electrochemical behavior of 2,6-TDA $(500 \mu \mathrm{M})$ on the surface of both bare and modified electrodes at pH 7.0 (B-R buffer) (Fig. 2).

The current response of the bare GCE was very weak, as demonstrated by an oxidation peak for 2,6-TDA of $0.079 \pm 0.008 \mu \mathrm{A}$ at $480 \mathrm{mV}$ (Fig. 2, trace a). Modification with MCNs did not show any significant improvement in the current response $(0.527 \pm 0.012 \mu \mathrm{A})$ compared to the bare electrode (Fig. 2, trace b), whereas a dramatic increase was measured after modification with MWCNTs, with an oxidation peak of $18.25 \pm 0.530 \mu \mathrm{A}$ (Fig. 2, trace c). This can be explained in consideration of the two-fold effect arising from the modification, that is, increased surface area and enhanced electron transfer rate of the electrode. As it is showed in Fig. 1A, the addition of MWCNTs led to a clear change in the surface topography, that is, the surface of the electrode exposed to the surrounding medium increased due to the tubular MWCNTs pointing out from the surface of the electrode. This explains the dramatic increase in the peak current, as reported before. To corroborate the positive effect of the increased active surface arising from the MWCNTs addition, it is possible to observe the outcome arising from the addition of MCNs
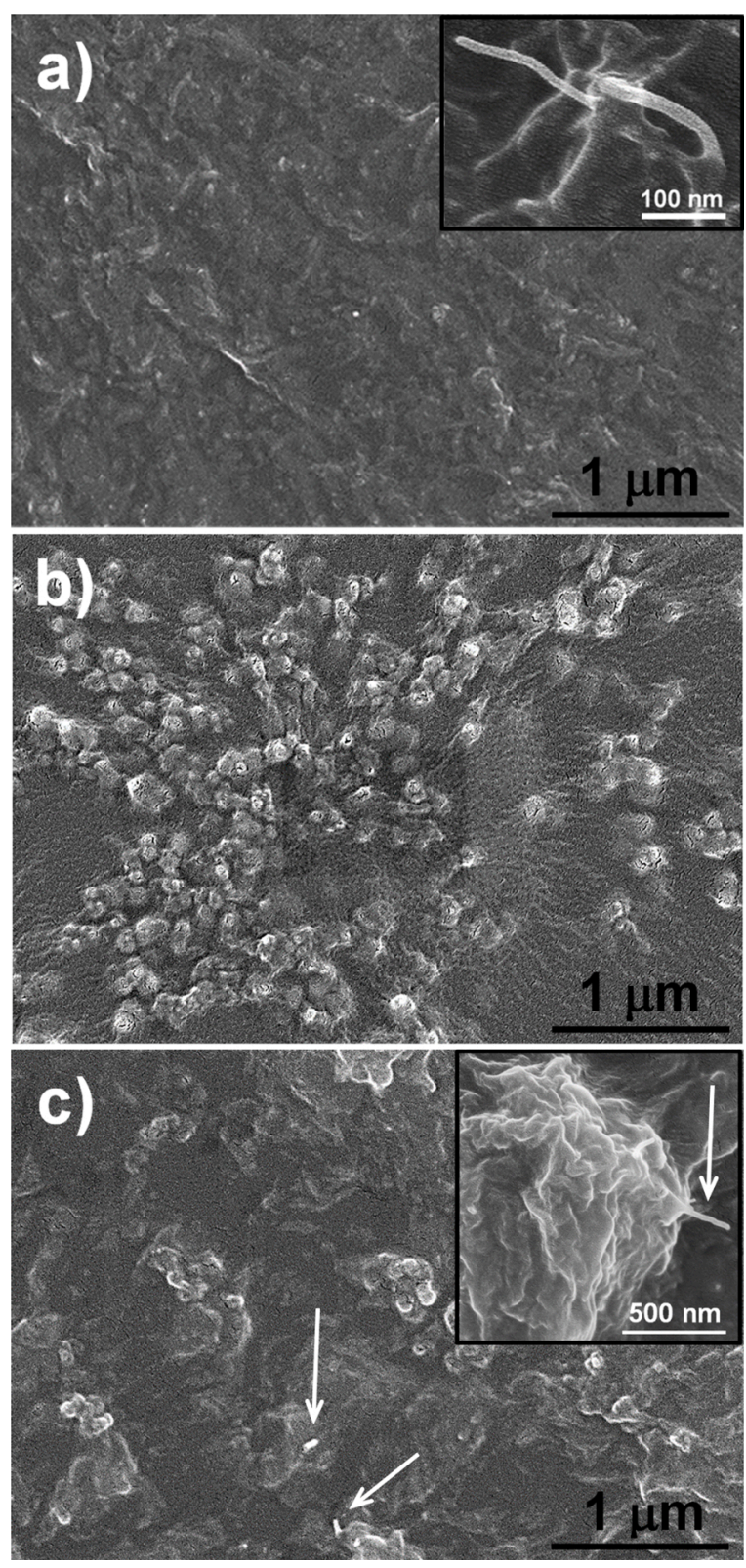

Fig. 1. FE-SEM surface images of (a) MWCNTs/GCE, (b) MCNs/GCE, and (c) MWCNTs-MCNs/GCE. The inset of panel (c) is a magnification of an individual MWCNT pointing from the modified electrode surface.

(Fig. 1B). In this case, although the surface topography changed significantly, the peak current did not increase greatly (Fig. 2, voltammogram b). This can be ascribed to the limited increase of the active electrode surface, which is plausibly due to the lower surface area of MCNs compared to the tubular MWCNTs. The electrochemical performance of the electrode was further improved using MWCNTs in combination with MCNs, with a maximum current response of $20.95 \pm 0.750$ $\mu$ A. As shown in Fig. 2 (trace d), the final output was slightly higher compared to the MWCNTs/GCE, most likely due to the MCNs' remarkable electronic properties, which eventually led to an overall increase electrode's conductivity.

\subsection{Effect of $p H$}

The $\mathrm{pH}$ of the environment surrounding the electrode surface may have an effect on the redox reaction that occurs between the analyte $(500 \mu \mathrm{M})$ and the modified-GCE. For this reason, we performed CV 


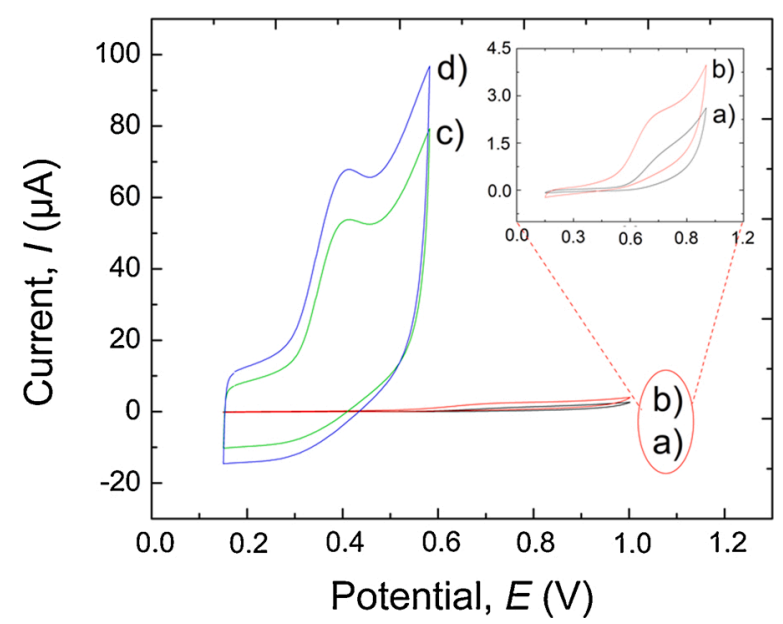

Fig. 2. Cyclic voltammograms in B-R buffer ( $\mathrm{pH} 7.0$ ) at a $50 \mathrm{mV} / \mathrm{s}$ scan rate of (from down-right to up-left direction) (a) bare GCE, (b) MCNs/GCE, (c) MWCNTs/GCE, and (d) MWCNTs-MCNs/GCE in the presence of $500 \mu \mathrm{M}$ TDA.

experiments over the $\mathrm{pH}$ range of $5.0-11$ in B-R buffer solution. As displayed in Fig. 3, the maximum oxidation peak current was reached at $\mathrm{pH}$ 7. Hence, all the following experiments were conducted at this $\mathrm{pH}$ value. It was also observed the linearly inverse relationship between oxidation peak potential and $\mathrm{pH}$, that is, increasing the $\mathrm{pH}$ led to a linear decrease of the oxidation peak potential (Fig. 3). As already noticed (Ghaani, Pucillo et al., 2018), this observation suggests that the oxidation reaction at the analyte/surface of the electrode interface plausibly involved protons, as corroborated by the linear relationship between the oxidation peak potential and the $\mathrm{pH}\left(\mathrm{E}_{\mathrm{pa}}=-11.084 \mathrm{pH}+475.55, \mathrm{R}^{2}\right.$ $=0.9518$ ). Finally, a negative shift of the potential by $11.084 \mathrm{mV}$ per $\mathrm{pH}$ unit was found.

\subsection{Influence of scan rate}

Information about an electrochemical mechanism can be obtained from the relationship between the peak current and scan rate. We thus investigated the influence of the scan rate on the electrocatalytic oxidation of 2,6-TDA at the MWCNT-MCN/GCE surface with CV within the $5-35 \mathrm{mVs}^{-1}$ range (analyte concentration of $500 \mu \mathrm{M}$ (Fig. 4). Each scan rate setting yielded an oxidation peak current $\left(I_{\mathrm{p}}\right)$. By plotting the oxidation peak current $\left(I_{\mathrm{p}}\right)$ generated by each scan rate setting against the square root of the scan rate, a linear relationship in the $5-35 \mathrm{mVs}^{-1}$ range was established (inset of Fig. 4). This observation suggests that at

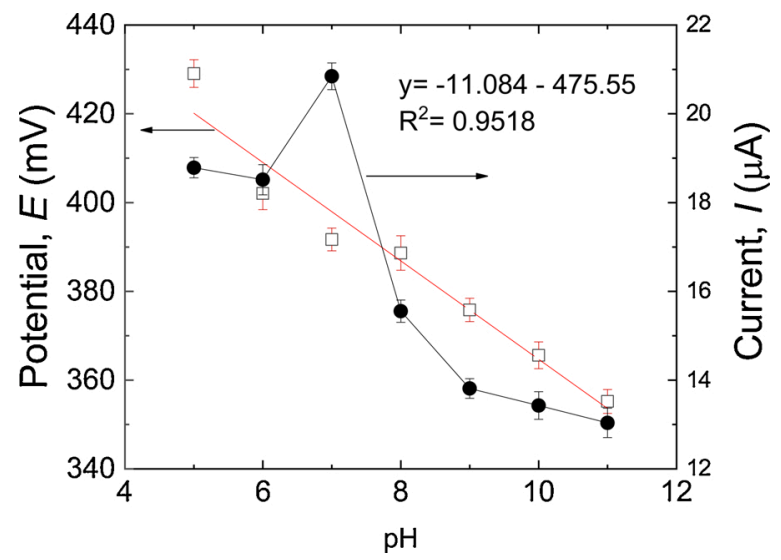

Fig. 3. Effect of $\mathrm{pH}$ (solution containing $500 \mu \mathrm{M}$ of 2,6-diaminotoluene in 0.1 $\mathrm{M}$ Britton-Robinson buffer) on the anodic peak potential $(E)$ and current $(I)$ of the MWCNTs-MCNs/GC electrode.

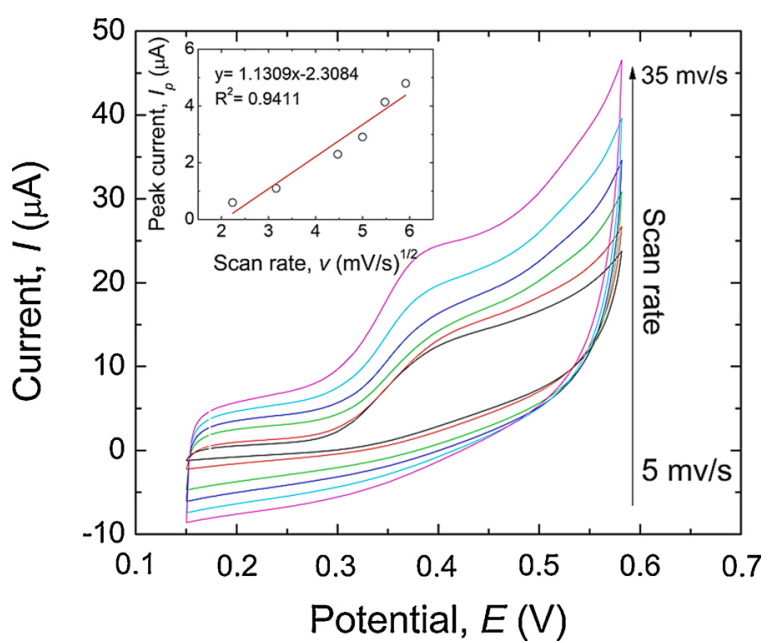

Fig. 4. Cyclic voltammograms of MWCNTs-MCNs/GCE in phosphate buffer (pH 7.0) containing $500 \mu \mathrm{M} 2,6-\mathrm{TDA}$ at different scan rates $\left(5-35 \mathrm{mVs}^{-1}\right)$. The electrocatalytic peak current $\left(I_{\mathrm{p}}\right)$ variation as a function of the square root of sweep rate is shown in the inset.

a sufficient overpotential the reaction is diffusion limited (Zare, Nasirizadeh, \& Mazloum Ardakani, 2005).

Running CV at different scan rates also allowed to achieve a better understanding of the kinetic mechanism of the GCE surface toward 2,6TDA. More specifically, applying the Laviron's theory it was possible to calculate the total number of electrons $(n)$ involved in the catalytic reaction using the following equation:

$E_{p a}=E^{0}+\left(\frac{R T}{\alpha n F}\right) \ln \left(\frac{R T K^{0}}{\alpha n F}\right)+\left(\frac{R T}{\alpha n F}\right) \ln v$

where $\alpha$ is the transfer coefficient, $K^{\circ}$ is the standard rate constant of the reaction, $n$ is the electron transfer number, $v$ is the scanning rate, $E^{\circ}$ is the formal redox potential, $R$ is the gas constant, $T$ is the absolute temperature, and $F$ is the faraday constant. Eq. (1), which applies for an irreversible electrode process, allowed to find a linear relationship between $E_{\mathrm{pa}}$ and $\ln (v)$, as expressed by the equation $E_{\mathrm{p}}(\mathrm{V})=0.0399 \ln (v)$ $\left(\mathrm{mVs}^{-1}\right)+0.2375$ (Fig. 5), starting from the raw voltammograms reported in Fig. 4. Using Laviron's equation it was thus possible to calculate the electron transfer number $(n)$, which is given by the slope of $E_{\mathrm{pa}}$ versus $\ln (v)$ (i.e., $\left.R T / \alpha n F\right)$. Finally, $n=1.17 \sim 1$ was obtained, which suggests that the electrochemical oxidation of 2,6-TDA at the MWCNT-

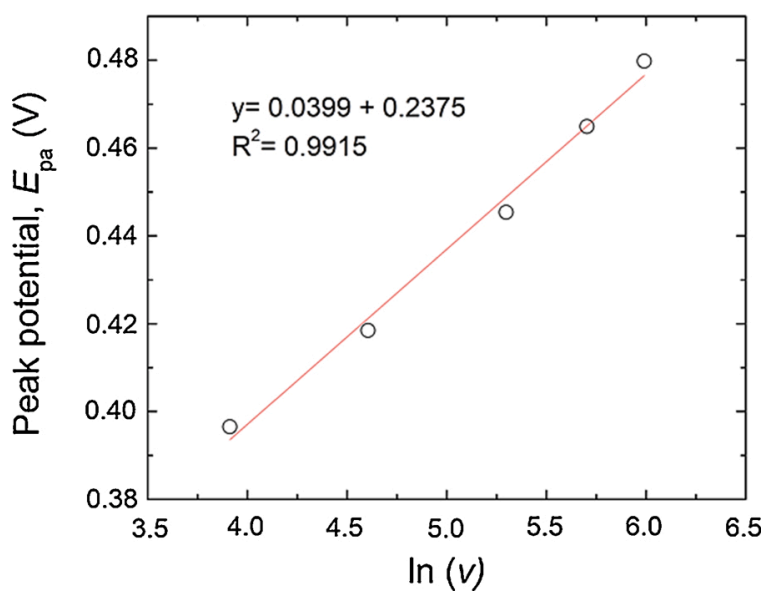

Fig. 5. Experimental data (empty circles) and linear regression of anodic peak potential (Epa) versus natural logarithm of the scan rate $[\ln (v)](50$ $400 \mathrm{mVs}^{-1}$ ). 
MCN/GCE surface is a one-electron transfer process. This finding differs from what was previously observed for other two PAAs, that is, 4,4'methylene diphenyl diamine and 2,4-diaminotoluene, for which the oxidation at the surface of a glassy carbon electrode modified with gold nanoparticles, chitosan, and multi-walled carbon nanotubes involved two electrons (Ghaani, Pucillo et al., 2018; Ghaani, Rovera et al., 2018).

\subsection{Chronoamperometry measurements}

Chronoamperometry experiments were performed to evaluate the electrochemical oxidation of 2,6-TDA. To this scope, chronoamperograms were obtained at a potential step of $480 \mathrm{mV}$, while the concentration of the analyte was varied between 0.04 and $5 \mathrm{mM}$ at $\mathrm{pH} 7$ (B-R buffer) (Fig. 6). Cottrell's equation was used to describe the current response of an electroactive material (such as 2,6-TDA) undergoing a diffusion-limited electrocatalytic process (Bard \& Faulkner, 2001):

$I=\frac{n F A D^{1 / 2} C_{b}}{\pi^{1 / 2} t^{1 / 2}}$

where $n$ is the electron transfer number of (1) exchanged per reactant molecule, $F$ is the Faraday constant $\left(9.648 \times 10^{4} \mathrm{Cmol}^{-1}\right), A$ is the geometric area of the electrode $\left(0.0653 \mathrm{~cm}^{2}\right)$, while $C_{\mathrm{b}}\left(\right.$ molcm $\left.{ }^{-3}\right)$, and $D\left(\mathrm{~cm}^{2} \mathrm{~s}^{-1}\right)$ are the concentration and the diffusion coefficient of 2,6TDA, respectively.

By plotting $I$ against $\mathrm{t}^{-1 / 2}$, different linear curves were obtained moving from $0.04 \mathrm{mM}$ to $5.0 \mathrm{mM} \mathrm{2,6-TDA} \mathrm{concentration} \mathrm{(Fig.} \mathrm{6,} \mathrm{inset} \mathrm{a).}$ The slope of each straight versus 2,6-TDA concentration finally made possible the calculation of the overall slope of the best-fit line (Fig. 6, inset b) using the following equation:

$I t^{1 / 2}=\frac{n F A D^{1 / 2} C_{b}}{\pi^{1 / 2}}$

From Eq. (3), $D$ can be extrapolated according to:

$D=\frac{(\text { slope })^{2} \pi}{\left(n F A C_{b}\right)^{2}}$

Finally, by using the overall slope within Cottrell's equation, an estimate of the average diffusion coefficient was obtained as $1.34 \times 10^{-4}$

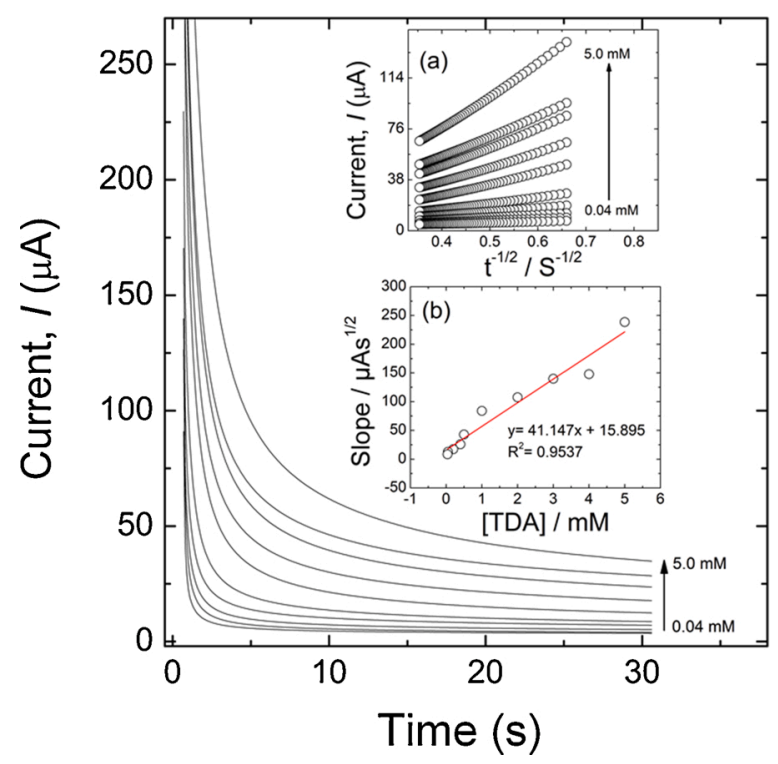

Fig. 6. Chronoamperograms obtained for the MWCNTs-MCNs/GCE in PBS (pH 7.0) at different concentrations of 2,6-TDA (0.04-5 mM). Insets: (a) plots of $I$ vs. $\mathrm{t}^{-1 / 2}$ obtained from the chronoamperograms (2,6-TDA concentration: 0.04-5 $\mathrm{mM}$ ); (b) plot of the slope of the straight lines against different 2,6-TDA concentrations. $\mathrm{cm}^{2} \mathrm{~s}^{-1}$

\subsection{Amperometric test}

Amperometry is more widely used than voltammetry to evaluate the sensitivity of a sensor owing to the different mechanism of transfer of the analyte to the electrode surface. Fig. 7 shows the amperometric response of a rotating MWCNTs-MCNs/GCE after the addition of 2,6-TDA at different concentrations (from 0.53-2326.60 $\mu \mathrm{M}$ ) into the $25 \mathrm{~mL} \mathrm{~B}-\mathrm{R}$ buffer at the potential step of $450 \mathrm{mV}$. A positive relationship occurred between the current response and the concentration of 2,6-TDA, with three main linear regions in three wide concentration ranges of 2,6-TDA: 0.53-11.37 $\mu \mathrm{M}$ (Fig. 7, inset a), 11.37-229.36 $\mu \mathrm{M}$ (Fig. 7, inset b), and 229.36-2326.60 $\mu \mathrm{M}$ (Fig. 7, inset c). The slope of the lower linear range in the amperometric plot was thus used to calculate the sensitivity of the MWCNTs-MCNs/GCE, which was $0.0232 \mu \mathrm{A}(\mu \mathrm{M})^{-1}$. In turn, the sensitivity of the modified electrode allowed calculating the lower limit of detection of 2,6-TDA, according to the following equation (Shrivastava \& Gupta, 2011; Skoog, Holler, \& Crouch, 2007):

$L O D=\frac{3 \times S_{b l}}{m}$

where $S_{\mathrm{bl}}$ is the standard deviation of the current response $(\mu \mathrm{A})$ obtained from the blank solution (10 replicates) and $m$ is the slope of the before mentioned lower linear range in the amperometric plot. Finally, the calculated LOD value for the MWCNTs-MCNs/GCE was $0.129 \mu \mathrm{M}$.

Amperometry experiments were also used to check for the operational stability of the MWCNT-MCN modified electrode. As displayed in the inset (d) of Fig. 7, no significant differences in the current response of the electrode were observed for $\sim 1280$ s in the B-R buffer containing 2,6-TDA. This observation supports the fact that there was no inhibitory effect of 2,6-TDA and its oxidation products on the modified electrode surface.

\subsection{Potential interference of other compounds}

The reliability of an electrochemical sensor in real systems can be

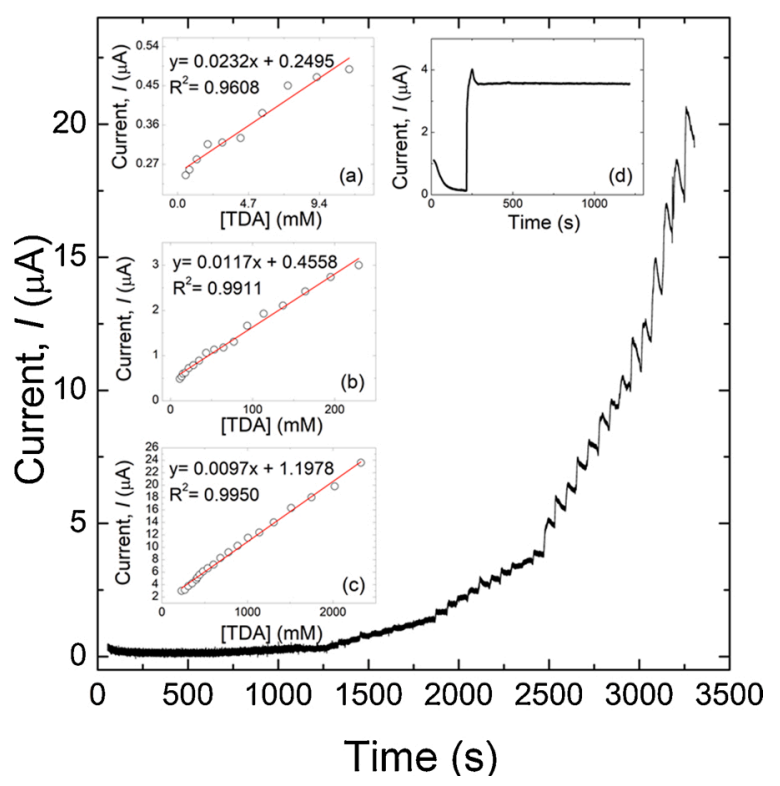

Fig. 7. Amperometric response at the rotating MWCNTs-MCNs/GCE at $480 \mathrm{mV}$ in $25 \mathrm{~mL}$ phosphate buffer $(\mathrm{pH} 7)(0.53-2326.60 \mu \mathrm{M})$. The variation of the amperometric current against the 2,6-TDA concentration is shown in insets (a) (0.53-11.37 $\mu \mathrm{M})$, (b) (11.37-229.36 $\mu \mathrm{M})$ (c) (229.36-2326.60 $\mu \mathrm{M})$. Inset (d) shows the stability of the response of the MWCNTs-MCNs/GCE for 1280s (2,6TDA concentration $550 \mu \mathrm{M})$. 
jeopardized due to interference phenomena, which are caused by the simultaneous presence of electroactive species other than the target analyte in the medium in contact with the electrode surface. If we consider a multilayer packaging material based on PU-adhesives, not only other PAAs, but also a number of additives commonly added during the extrusion process might impair the performance of the electrode due to interference with the target analyte. For this reason, in this work we decided to test the electrocatalytic performance of the MWCNT-MCN/ GCE toward 2,6-TDA in the presence of various substances as potentially interfering compounds.

At the beginning, aniline and 4,4'-MDA (two other PAAs) were tested. As shown if Fig. 8, the current response signal did not change when these two PAAs were added $(990 \mu \mathrm{M})$ to the solution containing 2,6-TDA, demonstrating that both compounds do not interfere with the detection of 2,6-TDA on the MWCNTs-MCNs/GC electrode surface. In a similar way, Irganox ${ }^{\circledR} 1010$ and Irgafos ${ }^{\circledR} 168$ (two additives used in the polyolefins manufacturing) were tested. Also in this case, the electrochemical performance of the MWCNT-MCN/GCE was not affected upon the addition of these two additives (200 $\mu \mathrm{M})$ (Fig. 8). The outcome of these tests proved that the MWCNT-MCN-modified electrode can be used to quantify selectively the 2,6-TDA.

\subsection{Real sample tests}

Real sample tests were carried out using the MWCNTs-MCNs/GCE to demonstrate its capacity for the detection and determination of 2,6-TDA that had possibly formed in packaging materials based on PU adhesives upon migration to the food. To this purpose, the food simulant B (acetic acid water solution, $3 \mathrm{w} / \mathrm{v} \%$ ) was used inside the thermo-sealed pouch, which was then exposed to $121^{\circ} \mathrm{C}$ for $20 \mathrm{~min}$ (sterilization conditions). The capability of the modified electrode to detect 2,6-TDA was assessed by the standard addition method (Sun \& Zhang, 2006). As shown in Table 1, the modified electrode the MWCNTs-MCNs/GCE performed decidedly well, with a high recovery of the analyte (97.42-106.54\%). This finding clearly indicates that the MWCNTs-MCNs/GCE can be efficiently used to detect the migration of 2,6-TDA that had possibly migrated from multilayer packages that undergo thermal stresses, such as sterilization, pasteurization, microwaving, and sous-vide cooking.

\subsection{Comparison of different analytical methods}

Many analytical methods have been developed for the detection and quantification of PAAs. The standard method developed by the Federal

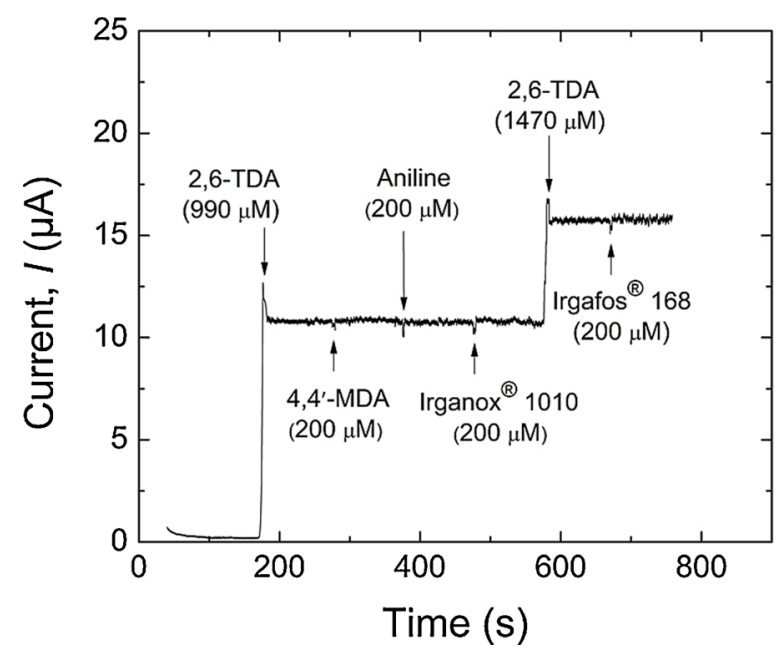

Fig. 8. Amperometric trace displaying the current response of the MWCNTsMCNs/GC electrode after the sequaential addition of the PAAs 2,6-TDA, 4,4'MDA, aniline and the two additives Irganox ${ }^{\circledR} 1010$ and $\operatorname{Irgafos}{ }^{\circledR} 168$.
Table 1

Amount of added (spiked) and measured 2,6-TDA at the MWCNTs-MCNs/GCE surface, with resulting recovery percentage after the migration test using the food simulant B under typical sterilization conditions $\left(121^{\circ} \mathrm{C}\right.$ for $\left.20 \mathrm{~min}\right)$.

\begin{tabular}{llll}
\hline Sample & Spiked $(\mu \mathrm{M})$ & Found $(\mu \mathrm{M})$ & Recovery $(\%)$ \\
\hline \multirow{3}{*}{ Sample 1 } & 0 & - & - \\
& 5.5 & 5.64 & 102.54 \\
& 35 & 37.29 & 106.54 \\
Sample 2 & 50 & 49.36 & 98.72 \\
& 0 & - & \\
& 5.5 & 5.42 & 98.54 \\
& 35 & 34.10 & 97.42 \\
Sample 3 & 50 & 50.83 & 101.66 \\
& 0 & - & 102 \\
& 5.5 & 5.61 & 99 \\
& 35 & 34.80 & 100 \\
\hline
\end{tabular}

German Institute for Consumer Protection and Food Safety is based on a spectrophotometric analysis, as previously reported. However, this method could overestimate PAAs since it determines the sum of all PAAs and can be affected by other substances such as colorants and impurities in the sample (Pezo, Fedeli, Bosetti, \& Nerín, 2012). To overcome these limitations, other analytical methods with or without preliminary sample preparation steps have been developed for the selective quantification of PAAs. In this context, Aznar et al. developed a method based on solid phase extraction (SPE) followed by LC-MS for the quantification of PAAs possibly migrated from multilayer films to the aqueous food simulant 3\% acetic acid (w/v) (Aznar, Canellas, \& Nerin, 2009). The high recovery range (81-109\%) indicated that this method was adequate for the application to real samples. Moreover, low detection limit, good linearity, and reproducibility were obtained as a result of the analysis. However, preliminary steps, including clean up and preconcentration, were time consuming, which eventually affected the overall efficiency of the procedure. Furthermore, the recovery of 2,6 TDA, which is one of the PAAs detected by this method, was as high as $93 \%$, which is lower than that obtained in our study. This method was modified for anaylzing eight primary aromatic amines (m-phenylenediamine, 2,6- and 2,4-toluenediamine, 1,5-diaminonaphthalene, aniline, 4,4' -diaminonaphenylether, 4,4' -methylenedianiline and 3,3' -dimethylbenzidine) without a clean up process. Even though high recovery and low detection limit were obtained by means of this modified method, many unknown compounds related to primary aromatic amines were also detected, which may cause false-positive results. In comparison to our study, 2,6 TDA was selectively detected and quantified by the MWCNTs/MCNs modified electrode in the presence of other compounds (Sendon, Bustos, Sánchez, Paseiro, \& Cirugeda, 2010). Mortensen et al. reported a method based on a LC/ESI-MS/MS apparatus, with no need for any preliminary step. The final results indicated an excellent accuracy in the determination of twenty primary aromatic amines related to polyurethane products and azo dyes (Mortensen et al., 2005). In another research, a HPLC-(SIM)-MS-based method has been developed to test plastic laminates and recycled paperboards for the migration of PAAs by Lambertini and co-workers (Lambertini et al., 2014). This method exhibited appropriate selectivity, sensitivity, repeatability, and a low detection limit of $0.1-3.6 \mu \mathrm{g} / \mathrm{kg}$. Even though the relevant method had proper sensitivity for the detection of twentytwo PAAs, 2,6 TDA was not amongst them. Brede et al. reported that solid phase derivatization as a pretreatment step for GC-MS analysis of possible PAAs migration from packaging materials in water food simulant provided low detection limit of $0.2 \mu \mathrm{g} / \mathrm{kg}$ for 2,6 TDA and good repeatability (Brede, Skjevrak, \& Herikstad, 2003). Besides, less time and solvent consuming represent other advantages of solid phase derivatization. On the other hand, although these methods have the advantage of high efficient separation, they are very expensive, time consuming, and highly specialized operators are needed to operate them. In our study, low limit of detection, high recovery of the analyte (97.42-106.54\%), excellent selectivity and 
stability have been reached by the MWCNTs/MCNs modified electrochemical sensor. Moreover, less time consuming, easiness to use, and relatively low cost of the MWCNTs/MCNs modified sensor makes this approach feasible for routine analysis in a standard packaging laboratory, especially for quality control.

\section{Conclusions}

In this work, we demonstrated how to modify the surface of a GCE to achieve adequate sensitivity and a low limit of detection for the determination of 2,6-TDA, a potential carcinogenic PAAs that can form as a NIAS in multilayer packaging materials whereby PU adhesives are used to join the different layers. The proposed modification of the working electrode surface was made using MWCNTs and MCNs, which acted in synergy (increased surface area and enhanced electroconductivity), eventually leading to the excellent electrocatalytic performance of the sensors. This was demonstrated by the outstanding sensitivity, detection limit, and stability of the sensor. Finally, the real samples experiments showed the capability of the MWCNTs-MCNs/GCE to provide reliable results as far as the quantification of 2,6-TDA is concerned. For all the above reasons, the modified sensor proposed in this work may represent an alternative analytical tool to commonly used analytical instrumentation, especially for quality control in industrial plants.

\section{Author statement}

Conceptualization: M.G., D.B. and S.F.; methodology, M.G. and D.B.; formal analysis, D.B. and M.G.; writing—original draft preparation, M. G. and D.B.; writing-review and editing, C.R., F.K. and S.F.; visualization, S.F.; supervision, S.F. All authors have read and agreed to the published version of the manuscript.

\section{References}

Aznar, M., Canellas, E., \& Nerín, C. (2009). Quantitative determination of 22 primary aromatic amines by cation-exchange solid-phase extraction and liquid chromatography-mass spectrometry. Journal of Chromatography A, 1216, 5176-5181. https://doi.org/10.1016/j.chroma.2009.04.096

Bard, A. J., \& Faulkner, L. R. (2001). Electrochemical methods: Fundamentals and applications (2nd ed.). John Wiley \& Sons, Inc.

Brauer, B., \& Funke, T. (1991). Determining primary aromatic-amines in migratory solutions. Deutsche Lebensmittel-Rundschau, 87, 280-281.

Brede, C., \& Skjevrak, I. (2004). Migration of aniline from polyamide cooking utensils into food simulants. Food Additives and Contaminants, 21, 1115-1124. https://doi. org /10.1080/02652030400019349

Brede, C., Skjevrak, I., \& Herikstad, H. (2003). Determination of primary aromatic amines in water food simulant using solid-phase analytical derivatization followed by gas chromatography coupled with mass spectrometry. Journal of Chromatography A, 983, 35-42. https://doi.org/10.1016/S0021-9673(02)01652-7

Cai, G., Ge, K., Ouyang, X., Hu, Y., \& Li, G. (2020). Thin-layer chromatography combined with surface-enhanced Raman scattering for rapid detection of benzidine and 4 aminobiphenyl in migration from food contact materials based on gold nanoparticle doped metal-organic framework. Journal of Separation Science, 43, 2834-2841. https://doi.org/10.1002/jssc.202000145

Campanella, G., Ghaani, M., Quetti, G., \& Farris, S. (2015). On the origin of primary aromatic amines in food packaging materials. Trends in Food Science \& Technology, 46, 137-143. https://doi.org/10.1016/j.tifs.2015.09.002

Chen, N., Chen, L., Cheng, Y., Zhao, K., Wu, X., \& Xian, Y. (2015). Molecularly imprinted polymer grafted graphene for simultaneous electrochemical sensing of 4, 4-methylene diphenylamine and aniline by differential pulse voltammetry. Talanta, 132, 155-161. https://doi.org/10.1016/j.talanta.2014.09.008

European Commission. (2011). Commission Regulation (EU), No. 10/2011 of 14 January. Official Journal of the European Union, 54, 1-89.

Ghaani, M., Pucillo, F., Olsson, R. T., Scampicchio, M., \& Farris, S. (2018). A bionanocomposite-modified glassy carbon electrode for the determination of 4,4' methylene diphenyl diamine. Analytical Methods, 10, 4122-4128. https://doi.org/ 10.1039/c8ay01376d

Ghaani, M., Rovera, C., Pucillo, F., Ghaani, M. R., Olsson, R. T., Scampicchio, M., et al. (2018). Determination of 2, 4-diaminotoluene by a bionanocomposite modified glassy carbon electrode. Sensors and Actuators B: Chemical, 277, 477-483. https:// doi.org/10.1016/j.snb.2018.09.053

Goulas, A. E., Riganakos, K. A., \& Kontominas, M. G. (2003). Effect of ionizing radiation on physicochemical and mechanical properties of commercial multilayer coextruded flexible plastics packaging materials. Radiation Physics and Chemistry, 68, 865-872. https://doi.org/10.1016/S0969-806X(03)00298-6

Hanssen, B. L., Siraj, S., \& Wong, D. K. (2016). Recent strategies to minimise fouling in electrochemical detection systems. Reviews in Analytical Chemistry, 35, 1-28. https:// doi.org/10.1515/revac-2015-0008

Lambertini, F., Di Lallo, V., Catellani, D., Mattarozzi, M., Careri, M., \& Suman, M. (2014). Reliable liquid chromatography-mass spectrometry method for investigation of primary aromatic amines migration from food packaging and during industrial curing of multilayer plastic laminates. Journal of Mass Spectrometry, 49, 870-877. https://doi.org/10.1002/jms.3436

Mattarozzi, M., Lambertini, F., Suman, M., \& Careri, M. (2013). Liquid chromatography-full scan-high resolution mass spectrometry-based method towards the comprehensive analysis of migration of primary aromatic amines from food packaging. Journal of Chromatography A, 1320, 96-102. https://doi.org/10.1016/j. chroma.2013.10.063

Mortensen, S. K., Trier, X. T., Foverskov, A., \& Petersen, J. H. (2005). Specific determination of 20 primary aromatic amines in aqueous food simulants by liquid chromatography electrospray ionization-tandem mass spectrometry. Journal of Chromatography A, 1091, 40-50. https://doi.org/10.1016/j.chroma.2005.07.026

Nasirizadeh, N., Shekari, Z., Zare, H. R., \& Makarem, S. (2013). Electrocatalytic determination of dopamine in the presence of uric acid using an indenedione derivative and multiwall carbon nanotubes spiked in carbon paste electrode. Materials Science and Engineering C, 33, 1491-1497. https://doi.org/10.1016/j. msec.2012.12.051

Nasirizadeh, N., Zare, H. R., Fakhari, A. R., Ahmar, H., Ahmadzadeh, M. R., \& Naeimi, A. (2011). A study of the electrochemical behavior of an oxadiazole derivative electrodeposited on multi-wall carbon nanotube-modified electrode and its application as a hydrazine sensor. Journal of Solid State Electrochemistry, 15, 2683-2693. https://doi.org/10.1007/s10008-010-1259-6

Pezo, D., Fedeli, M., Bosetti, O., \& Nerín, C. (2012). Aromatic amines from polyurethane adhesives in food packaging: The challenge of identification and pattern recognition using Quadrupole-Time of Flight-Mass Spectrometry ${ }^{\mathrm{E}}$. Analytica Chimica Acta, 756, 49-59. https://doi.org/10.1016/j.aca.2012.10.031

Rassaei, L., Marken, F., Sillanpää, M., Amiri, M., Cirtiu, C. M., \& Sillanpää, M. (2011). Nanoparticles in electrochemical sensors for environmental monitoring. TrAC Trends in Analytical Chemistry, 30, 1704-1715. https://doi.org/10.1016/j.trac.2011.05.009

Sanchis, Y., Coscollà, C., \& Yusà, V. (2019). Comprehensive analysis of photoinitiators and primary aromatic amines in food contact materials using liquid chromatography High-Resolution Mass Spectrometry. Talanta, 191, 109-118. https://doi.org/ 10.1016/j.talanta.2018.08.047

Sendon, R., Bustos, J., Sánchez, J. J., Paseiro, P., \& Cirugeda, M. E. (2010). Validation of a liquid chromatography-mass spectrometry method for determining the migration of primary aromatic amines from cooking utensils and its application to actual samples. Food Additives and Contaminants, 27, 107-117. https://doi.org/10.1080/ 02652030903225781

Shrivastava, A., \& Gupta, V. B. (2011). Methods for the determination of limit of detection and limit of quantitation of the analytical methods. Chronicles of Young Scientists, 2, 21.

Skoog, D. A., Holler, F. J., \& Crouch, S. R. (2007). Principles of instrumental analysis. Canada: Thomson Brooks. Cole.

Sun, D., \& Zhang, H. (2006). Voltammetric determination of 6-benzylaminopurine (6BAP) using an acetylene black-dihexadecyl hydrogen phosphate composite film coated glassy carbon electrode. Analytica Chimica Acta, 557, 64-69. https://doi.org/ 10.1016/j.aca.2005.10.002

Ubeda, S., Aznar, M., Rosenmai, K., Vinggaard, A. M., \& Nerín, C. (2020). Migration studies and toxicity evaluation of cyclic polyesters oligomers from food packaging adhesives. Food Chemistry, 311, 125918-125928. https://doi.org/10.1016/j. foodchem. 2019.125918

Vineis, P., \& Pirastu, R. (1997). Aromatic amines and cancer. Cancer Causes \& Control, 8, 346-355. https://doi.org/10.1023/A:1018453104303

Viswanathan, S., \& Radecki, J. (2008). Nanomaterials in electrochemical biosensors for food analysis-a review. Polish Journal of Food and Nutrition Sciences, 58, 157-164.

Wang, H.-B., Zhang, H.-D., Xu, L.-L., Gan, T., Huang, K.-J., \& Liu, Y.-M. (2014). Electrochemical biosensor for simultaneous determination of guanine and adenine based on dopamine-melanin colloidal nanospheres-graphene composites. Journal of Solid State Electrochemistry, 18, 2435-2442. https://doi.org/10.1007/s10008-0142494-Z

Wang, H.-B., Zhang, H.-D., Zhang, Y.-H., Chen, H., Xu, L.-L., Huang, K.-J., et al. (2015). Tungsten disulfide nano-flowers/silver nanoparticles composites based electrochemical sensor for theophylline determination. Journal of the Electrochemical Society, 162, 173-179. https://doi.org/10.1149/2.0941507jes

Yan, J. W., Hu, C., Tong, L. H., Lei, Z. X., \& Lin, Q.-B. (2020). Migration test and safety assessment of polyurethane adhesives used for food-contact laminated films. Food Packaging and Shelf Life, 23, 100449-100456. https://doi.org/10.1016/j. fpsl.2019.100449

Zare, H. R., Nasirizadeh, N., \& Mazloum Ardakani, M. (2005). Electrochemical properties of a tetrabromo-p-benzoquinone modified carbon paste electrode. Application to the simultaneous determination of ascorbic acid, dopamine and uric acid. Journal of Electroanalytical Chemistry, 577, 25-33. https://doi.org/10.1016/j. jelechem.2004.11.010

Zhang, H., Xing, Z., Pan, M., Wang, H.-B., \& Liu, Y.-M. (2020). Highly sensitive and selective electrochemical determination of 4-aminophenol based on flower-like AgAu nanocomposites modified glassy carbon electrode. Journal of the Electrochemical Society, 167, 126504-126511. https://doi.org/10.1149/1945-7111/abae91 